\title{
Incidence and Predictors of Acute Kidney Injury Among Women With Severe Pre-eclampsia at Mbarara Regional Referral Hospital
}

\author{
Mariam Hassan \\ Mbarara University of Science and Technology \\ Roland Mayanja \\ Mbarara University of Science and Technology \\ Wasswa G.M Ssalongo \\ Mbarara Regional Referral Hospital, Uganda \\ Natumanya Robert \\ Mbarara Regional Referral Hospital, Uganda \\ Lugobe Henry Mark \\ Mbarara University of Science and Technology \\ Okello Samson \\ Mbarara University of Science and Technology \\ Rose Muhindo ( $\nabla$ drmuhindo@gmail.com ) \\ Mbarara University of Science and Technology
}

\section{Research Article}

Keywords: severe pre-eclampsia, eclampsia, acute kidney injury

Posted Date: November 29th, 2021

DOI: https://doi.org/10.21203/rs.3.rs-1093153/v1

License: (9) This work is licensed under a Creative Commons Attribution 4.0 International License. Read Full License 


\section{Abstract}

\section{Background}

The presence of acute kidney injury (AKI) in pre-eclampsia complicates treatment including; increasing length of hospital stay and a need to access services like dialysis which are largely expensive in resourcelimited settings. We aimed to determine incidence and predictors of acute kidney injury among women with severe pre-eclampsia at Mbarara Regional Referral Hospital in southwestern Uganda.

\section{Methods}

We carried out a hospital-based prospective cohort study from 16 November 2018 to 18 April 2019, among pregnant women with severe preeclampsia followed up in the hospital. We enrolled 70 mothers with severe pre-eclampsia and eclampsia; we excluded patients with a history of chronic renal disease, chronic hypertension, and gestational hypertension.

Data on socio-demographics, laboratory parameters, health system, obstetric and medical factors were collected. Baseline serum creatinine, complete blood count, and CD4 T-cell count were all done at admission (0-hour). Second serum creatinine was done at 48-hours to determine the presence of AKI. AKI was defined as a rise in serum creatinine of $0.3 \mathrm{mg} / \mathrm{dl}$ or more from the baseline. The proportion of women diagnosed with acute kidney injury among the total number of women with severe pre-eclampsia was reported as incidence proportion. Univariate and multivariate logistic regression was used to establish the association of acute kidney injury and severe pre-eclampsia.

Results

Incidence of acute kidney injury was high (41.4\%) among women with severe pre-eclampsia. Antenatal care attendance was protective $0.36(0.16,0.80), \mathrm{p}<0.013$ at bivariate analysis but had no statistical significance at multivariate analysis. Eclampsia was an independent risk factor for acute kidney injury. (aRR $2.74(1.06,7.08), \mathrm{P}<0.037$.

Conclusion

The incidence of acute kidney injury in patients with preeclampsia is high.

Eclampsia is an independent risk factor of acute kidney injury.

\section{Introduction}

Acute Kidney Injury (AKI) is defined as an abnormality of the excretory function of the kidney characterized by an accumulation of nitrogenous waste which includes urea and creatinine(1). AKI normally happens in hours, days, or weeks and is potentially reversible. The diagnosis of AKI is normally made on history, measurement of kidney function, and detection of urine abnormalities. 
Incidence and factors associated with acute kidney injury in women with severe pre-eclampsia vary. Severe renal impairment occurs frequently when pre-eclampsia is complicated by abruption placentae or hemolysis, elevated liver enzymes, and low platelet count (HELLP) syndrome (2). A history of preeclampsia in previous pregnancies increases the risk of acute kidney injury by 3 while this risk is increased to 5 if two or more previous pregnancies were complicated by pre-eclampsia(3).

The most common causes of AKI in pregnancy are preeclampsia and eclampsia, which can ultimately lead to an increase in maternal mortality rates(4). In Africa, AKI is a challenging problem because of the burden of disease, the late presentation of patients to health care facilities, and the lack of resources to support patients with established AKI in many countries(5).

The presence of AKI in pre-eclampsia complicates the treatment including increasing the length of hospital stay, the need to access services like dialysis which are largely expensive. It is therefore paramount to know the incidence and predictors of AKI in the setting of Mbarara Regional Referral Hospital in Southwestern Uganda in mothers admitted with severe pre-eclampsia.

\section{Study Design, Setting And Population:}

We prospectively enrolled 70 participants on the maternity ward at Mbarara Regional Referral Hospital (MRRH) to determine the incidence and predictors of acute kidney injury in mothers admitted with severe pre-eclampsia. MRRH is a public regional referral hospital that serves as a teaching hospital for Mbarara University of Science and Technology. The hospital serves as a referral for the western region of Uganda but also receives patients from refugee camps of Rwanda, Congo, and Burundi. The hospital offers specialized services, employs eleven obstetricians and 32 midwives together with resident doctors who perform over 10,000 deliveries annually. The hospital also can perform laboratory tests including chemistries, urinalysis, and ultrasonography among others. A resident nephrologist in the hospital can perform acute hemodialysis for patients who require the service.

The study population consisted of all women having severe pre-eclampsia and eclampsia between November 2018 to April 2019. We included all women who were diagnosed with severe preeclampsia/eclampsia defined as high blood pressure $(\geq 140 / 90 \mathrm{mmHg})$ plus one or more of the following symptoms of severity (headache, epigastric pain, blurring of vision, convulsions, and proteinuria plus 2 or 3 on urine dipstick). Women who had a history or documentation of chronic renal disease and chronic hypertension and gestational hypertension were excluded. Eligible women were recruited until the sample size was attained. Consent was obtained and blood samples were taken for baseline renal function tests. After 48 hours another sample was drawn for a 2 nd creatinine. A difference of serum creatinine $\geq$ $0.3 \mathrm{mg} / \mathrm{dl}$ from the baseline was considered $\mathrm{AKI}$ and patients were followed up by the study team while in hospital from the time of admission to when they were discharged. The sample size was estimated based on the Fleiss formula (1981) for cohort studies.

The major outcome variable of the study was acute kidney injury. The primary exposure variable was severe pre-eclampsia while the secondary exposure variables were sociodemographic factors, healthcare 
system factors, obstetric factors which included gravidity/parity, gestation age at diagnosis of preeclampsia and admission to the hospital, postpartum hemorrhage, antepartum hemorrhage, eclampsia, HELLP syndrome and previous history of pre-eclampsia. The medical factors were a history of diabetes, hypertension, and HIV infection.

\section{Statistical analysis}

Data was captured using Redcap, and exported to Excel. All analyses were performed using Stata software Version 13.0. Continuous variables such as age were presented as means and standard deviation for normally distributed variables or median and interquartile range for variables not normally distributed. The incidence proportion of AKI was derived as the proportion of women diagnosed with acute kidney injury among the total number of women with severe pre-eclampsia/eclampsia. Bivariate and multivariate logistic regression models were used to identify predictors of acute kidney injury. Predictor variables were variables with relative risk. Additional variables were included if on bivariate analysis they demonstrated a correlation with the outcome of interest (acute kidney injury) with a $P$-value $<0.05$. Backward stepwise elimination was used to create the final model, and all variables with $P$ values $<0.05$ in the final model were considered significant independent factors associated with acute kidney injury.

\section{Results}

A total of 4547 obstetrics and gynecology admissions were conducted during the study period, out of which 75 met the inclusion criteria. Among those eligible, some participants refused to participate while others escaped from the hospital before official discharge. 70 participants were recruited.

The mean age of our study participants was $27.5( \pm 6.4)$ years with $87.1 \%$ of them being married. $65.7 \%$ of the participants were housewives and $76.7 \%$ of the participants were referred from lower health facilities. A majority (85.3\%) reported having attended antenatal care clinics. The commonest gestational age at delivery was 36 weeks and above. HELLP syndrome accounted for $27.1 \%$ of cases while $21.4 \%$ had eclampsia. The mean systolic blood pressure was $166( \pm 28.5)$ while the diastolic was $108( \pm 16.8) \mathrm{mmHg}$. The mean baseline creatinine was $0.9 \mathrm{mg} / \mathrm{dl}( \pm 0.3) .68 .6 \%$ of the participants had proteinuria and the mean hemoglobin level was $11.3 \mathrm{~g} / \mathrm{dl}( \pm 2.4)$. The details of the participants' baseline characteristics are shown in

\section{Table 1: Baseline characteristics of study participants}




\begin{tabular}{|c|c|}
\hline Characteristics & $\begin{array}{l}\text { Overall } N=70 \\
N(\%)\end{array}$ \\
\hline Age, in years, mean (SD) & $27.5( \pm 6.4)$ \\
\hline \multicolumn{2}{|l|}{ Education category } \\
\hline None & $26(37.1)$ \\
\hline Primary & $20(28.6)$ \\
\hline \multicolumn{2}{|l|}{ Marital status } \\
\hline Married & $61(87.1)$ \\
\hline Housewife & $46(65.7)$ \\
\hline \multicolumn{2}{|l|}{ Referring facility } \\
\hline HC III and IV & $39(76.5)$ \\
\hline \multicolumn{2}{|l|}{ Obstetric factors } \\
\hline Multigravida & $51(72.9)$ \\
\hline ANC attendance, yes $(N=68)$ & $58(85.3)$ \\
\hline \multicolumn{2}{|l|}{ Gestational age at diagnosis } \\
\hline 20 - 28 weeks & $12(17.1)$ \\
\hline 28 - 34 weeks & $18(25.7)$ \\
\hline $34-36$ weeks & $12(17.1)$ \\
\hline 36 weeks $\&$ above & $28(40.0)$ \\
\hline Abruption placenta & $4(5.7)$ \\
\hline HELLP syndrome & $19(27.1)$ \\
\hline Eclampsia & $15(21.4)$ \\
\hline \multicolumn{2}{|l|}{ Laboratory parameters } \\
\hline Haemoglobin in g/dl, mean (SD) & $11.3( \pm 2.4)$ \\
\hline$<10 \mathrm{~g} / \mathrm{dl}$ & $13(18.6)$ \\
\hline$>10 \mathrm{~g} / \mathrm{dl}$ & $57(81.4)$ \\
\hline Proteinuria & $48(68.6)$ \\
\hline Baseline creatinine at admission in $\mathrm{mg} / \mathrm{dl}$, mean (SD) & $0.90( \pm 0.3)$ \\
\hline HIV status, positive & $5(7.1)$ \\
\hline
\end{tabular}


Abbreviations: HC Health Center, ANC Antenatal Care, HIV Human Immunodeficiency Virus, HELLPHemolysis Elevated Liver Enzymes Low platelets, SBP Systolic Blood pressure, DBP Diastolic Blood pressure

\section{Incidence of Acute Kidney Injury}

The incidence of acute kidney injury among women with severe pre-eclampsia/ eclampsia was 29/70 $(41.4 \%)$ and is diagrammatically represented in figure 1.

\section{Predictors of acute kidney injury in logistic regression (both univariate multivariate analysis)}

Antenatal care attendance was protective with a crude risk ratio of $0.36(0.16,0.80), p<0.013$ in bivariate analysis but there was no statistical significance at multivariate analysis. Eclampsia was an independent risk factor of acute kidney injury (aRR $2.74,95 \% \mathrm{Cl} 1.06-7.08, \mathrm{P}<0.037$ ). The details of the predictors of acute kidney injury among women with pre-eclampsia are shown in table 2.

\section{Table 2: Predictors of acute kidney injury by logistic regression}

Abbreviations: ANC Antenatal Care, HIV Human Immunodeficiency Virus, SBP Systolic Blood Pressure, DBP Diastolic Blood Pressure

\section{Discussion}

In this prospective study, our main goal was to establish the incidence and predictors of AKI among mothers admitted with pre-eclampsia admitted at the maternity ward of Mbarara Regional Referral Hospital (MRRH). We found a high cumulative incidence of AKI of $41.4 \%$, and eclampsia as the main independent predictor of AKI, within 48 hours after admission. Our findings were lower than what was reported among severe preeclampsia/eclampsia patients in Cameroon among whom $66.7 \%$ developed acute kidney injury. This could be because a majority ( $72.5 \%$ ) of the enrolled participants in the study done in Cameroon had eclampsia (6) compared to our study which had only $21.4 \%$ patients with eclampsia. Furthermore, the Cameroon study was conducted in an intensive care unit and also had 7 years of follow-up. Data from developed countries suggests lower rates of AKI, with estimates as low as 1-2.8\% (7). However, in developing countries, the incidence of AKI is high ranging from $36 \%$ to $66.7 \%(8,9)$, Cameroon $(66.7 \%)(10)$, Morocco $(66.7 \%)(11)$. The high incidence of AKI in developing countries may be attributed to the late presentation of patients to hospitals for care.

In our study eclampsia was the main independent predictor of AKI (aRR 2.74, (5\% Cl $1.06-7.08$, 


\begin{tabular}{|c|c|c|c|c|}
\hline \multirow[t]{2}{*}{ Characteristic } & Bivariate analysis & p-value & Multivariate analysis & P-value \\
\hline & \multicolumn{2}{|l|}{ CRR (95\%Cl) } & \multicolumn{2}{|l|}{ aRR (95\%Cl) } \\
\hline Age (each year) & $1.022(0.97,1.08)$ & 0.444 & $1.00(0.90,1.11)$ & 0.991 \\
\hline \multicolumn{5}{|l|}{ ANC attendance } \\
\hline No & Ref & & Ref & \\
\hline Yes & $0.36(0.16,0.80)$ & 0.013 & $0.49(0.16,1.54)$ & 0.221 \\
\hline \multicolumn{5}{|l|}{ Eclampsia } \\
\hline No & Ref & & Ref & \\
\hline Yes & $2.24(1.06,474)$ & 0.035 & $2.74(1.06,7.08)$ & 0.037 \\
\hline \multicolumn{5}{|l|}{ HIV status } \\
\hline Negative & Ref & & Ref & \\
\hline Positive & $1.95(0.26,14.43)$ & 0.514 & $2.01(0.26,15.62)$ & 0.506 \\
\hline \multicolumn{5}{|c|}{$S B P>160$ or $D B P>110$} \\
\hline No & Ref & & Ref & \\
\hline Yes & $1.27(0.59,2.72)$ & 0.545 & $2.41(0.81,7.18)$ & 0.115 \\
\hline \multicolumn{5}{|l|}{ Gravidity } \\
\hline 2 or less & Ref & & & \\
\hline 3 or more & $1.51(0.70,3.25)$ & 0.291 & $0.87(0.11,6.75)$ & 0.897 \\
\hline \multicolumn{5}{|l|}{ Parity } \\
\hline 2 or less & Ref & & & \\
\hline 3 or more & $1.94(0.92,4.11)$ & 0.381 & $1.83(0.31,10.96)$ & 0.510 \\
\hline \multicolumn{5}{|c|}{ Gestational age at delivery } \\
\hline $20-28$ & Ref & & Ref & \\
\hline $28-34$ & $0.47(0.13,1.61)$ & 0.228 & $0.72(0.13,3.93)$ & 0.708 \\
\hline 34 or more & $0.64(0.29,1.43)$ & 0.281 & $0.74(0.23,2.31)$ & 0.600 \\
\hline
\end{tabular}

$\mathrm{P}<0.037)$. This is in agreement with a prospective hospital-based study done in India which revealed that acute kidney injury was common among patients with eclampsia as high as $25-50 \%$ (12). This may be so because pre-eclampsia and eclampsia are associated with glomerular endotheliosis which decreases the glomerular filtration rate and renal blood flow. An increase in renal vascular resistance predisposes patients to AKI which is characterized by proteinuria and renal failure. 
In our study, we noted that antenatal care attendance was associated with a reduction in the incidence of acute kidney injury compared to non-attendance; crude risk ratio 0.36, 95\% C.I, 0.16-0.80, p-value 0.013. However, this was not significant at multivariate analysis and there was no association between the number of times the mothers attended antenatal care and the incidence of AKI. There is evidence to suggest that women with severe pre-eclampsia who present with severe complications are likely to have attended ANC fewer times than those without major complications (Manandhar et al., 2013). This may be so because antenatal attendance is associated with early detection of disease and timely intervention due to the structured routine screening of blood pressure and urine protein during antenatal visits.

However, some studies found no association between the number of times of antenatal care attendance and the severity of pre-eclampsia on presentation $(13,14)$. This may be because the disease progression pattern is most times unpredictable and acute complications can occur without warning symptoms.

The inability to use measure more sensitive renal biomarkers for the diagnosis of AKI was one of our study limitations. Serum creatinine takes time to rise and may lead to late detection of AKI.

Urine output is not routinely monitored quantitatively unless in very sick or catheterized patients.

\section{Conclusion}

The incidence of acute kidney injury in patients with pre-eclampsia/eclampsia is high (41.4\%).

The presence of eclampsia in patients admitted with severe pre-eclampsia is associated with the development of AKI within 48 hours after hospitalization. We recommend a larger prospective study that will include the use of biomarkers for acute kidney injury and closer monitoring and management of mothers with eclampsia for features of acute kidney injury.

\section{Abbreviations}

AKI: $\quad$ Acute Kidney Injury

ALP: $\quad$ Alkaline phosphatase

AST: $\quad$ Aspartate aminotransferase

ALT: $\quad$ Alanine aminotransferase

ATN: $\quad$ Acute tubular necrosis

CBC: $\quad$ Complete blood cell count

CKD: $\quad$ Chronic kidney diseases

eGFR: $\quad$ Estimated Glomerular filtration rate 
HELLP: Hemolysis, elevated liver enzymes and low platelet syndrome
LFT:
Liver function test

MRRH: Mbarara Regional Referral Hospital

MUST: $\quad$ Mbarara University of science and Technology

OBS/GYN: Obstetrics and Gynecology

RFT: $\quad$ Renal function test

\section{Declarations}

\section{Author Details}

Mariam Hassan ${ }^{1}$, Roland Mayanja* ${ }^{* 1}$, Wasswa G.M Ssalongo ${ }^{2}$, Natumanya Robert ${ }^{3}$, Lugobe Henry Mark ${ }^{1}$, Okello Samson², Rose Muhindo*2

1- Department of obstetrics and gynecology, Mbarara University of Science and Technology, Uganda

2- Department of Internal Medicine, Mbarara Regional Referral hospital

3- $\quad$ Mbarara Regional Referral Hospital

\section{Acknowledgments}

We thank the pregnant mothers who accepted to be part of our study.

\section{Author contributions}

All authors named in this manuscript have contributed substantially to this work and meet the criteria for authorship. MH, RM and RM took part in initial concept design, proposal writing, data collection, data interpretation, manuscript writing/revision, and approval of final work.

GW, SO, HML contributed towards data interpretation, revision of the manuscript, and approval of final work. NR contributed towards manuscript writing/revision.

\section{Competing interests}

The authors declare that they have no competing interests.

\section{Ethics approval and Informed consent}


This study was approved by the Mbarara University of Science and Technology's faculty review board and institutional ethics review board, and Uganda national council for science and technology. We confirm that all eligible participants or their caregivers provided informed consent to participate in this study and that the study got approval from the necessary bodies. All methods in this study were carried out in accordance with relevant guidelines and regulations.

Consent to publish: Not applicable.

Availability of data and materials: The datasets used during this study are available from the corresponding author upon reasonable request.

Source of Funding: The authors did not receive funding for this study

\section{References}

1. Bellomo R, Kellum JA, Ronco C. Acute kidney injury. The Lancet. 2012;380(9843):756-66.

2. Drakeley AJ, Le Roux PA, Anthony J, Penny J. Acute renal failure complicating severe preeclampsia requiring admission to an obstetric intensive care unit. American journal of obstetrics and gynecology. 2002;186(2):253-6.

3. Vikse BE, Irgens LM, Leivestad T, Skjærven R, Iversen BM. Preeclampsia and the risk of end-stage renal disease. New England Journal of Medicine. 2008;359(8):800-9.

4. Wattanavaekin K, Kitporntheranunt M, Kreepala C. Cystatin $\mathrm{C}$ as a novel predictor of preterm labor in severe preeclampsia. Kidney research and clinical practice. 2018;37(4):338.

5. Naicker S, Aboud O, Gharbi MB. Epidemiology of Acute Kidney Injury in Africa. Seminars in Nephrology.28(4):348-53.

6. Priso EB, Njamen TN, Tchente CN, Kana AJ, Landry T, Tchawa UFN, et al. Trend in admissions, clinical features and outcome of preeclampsia and eclampsia as seen from the intensive care unit of the Douala General Hospital, Cameroon. Pan African Medical Journal. 2015;21(1).

7. Aggarwal RS, Mishra VV, Jasani AF, Gumber M. Acute renal failure in pregnancy: our experience. Saudi Journal of Kidney Diseases and Transplantation. 2014;25(2):450.

8. Arrayhani M, El Youbi R, Sqalli T. Pregnancy-related acute kidney injury: experience of the nephrology unit at the university hospital of fez, morocco. ISRN nephrology. 2012;2013.

9. Prakash J. The kidney in pregnancy: A journey of three decades. Indian journal of nephrology. 2012;22(3):159-67. 
10. Priso EB, Njamen TN, Tchente CN, Kana AJ, Landry T, Niaga F, et al. Trend in admissions, clinical features and outcome of preeclampsia and eclampsia as seen from the intensive care unit of the Douala General Hospital, Cameroon. Pan African Medical Journal. 2015;21.

11. Ansari MR, Laghari MS, Solangi KB. Acute renal failure in pregnancy: one year observational study at Liaquat University Hospital, Hyderabad. JPMA The Journal of the Pakistan Medical Association. 2008;58(2):61.

12. Mjahed K, Alaoui SY, Barrou L. Acute renal failure during eclampsia: incidence risks factors and outcome in intensive care unit. Renal failure. 2004;26(3):215-21.

13. Manandhar BL, Chongstuvivatwong V, Geater A. Antenatal care and severe pre-eclampsia in Kathmandu valley. Journal of Chitwan Medical College. 2013;3(6):43-7.

14. Yakasai IA, Morhason-Bello IO. Risk factors for pre-eclampsia among women at antenatal booking in Kano, Northern Nigeria. Healthcare in Low-resource Settings. 2013;1(1):12.

\section{Figures}

\section{INCIDENCE OF ACUTE KIDNEY INJURY}

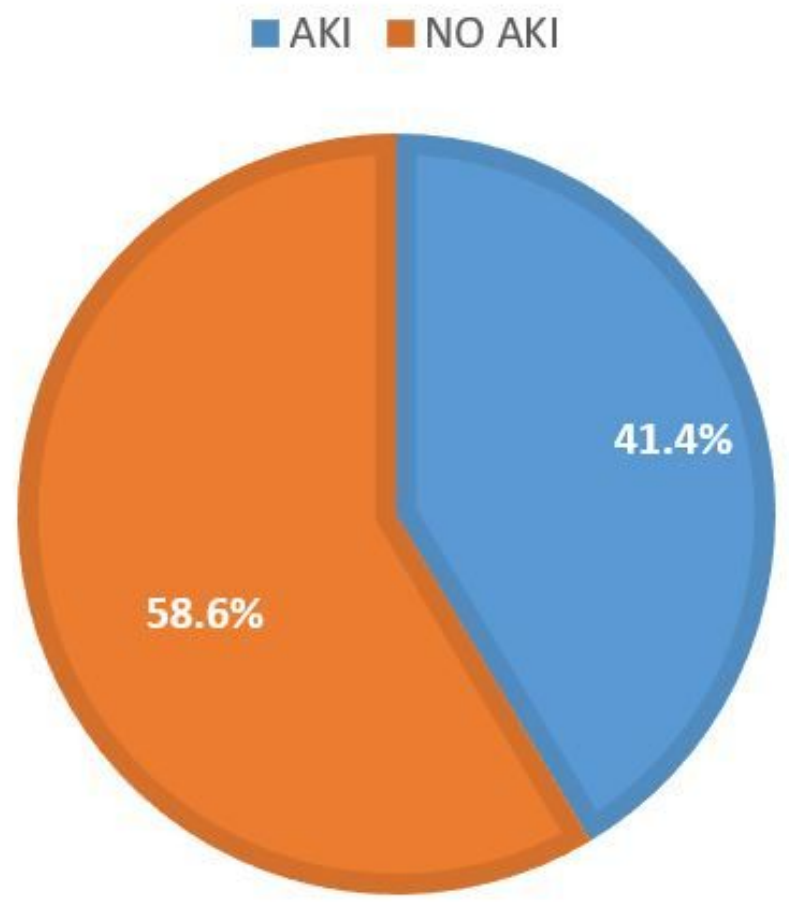

\section{Figure 1}

Incidence of acute kidney injury among mothers with pre-eclampsia 